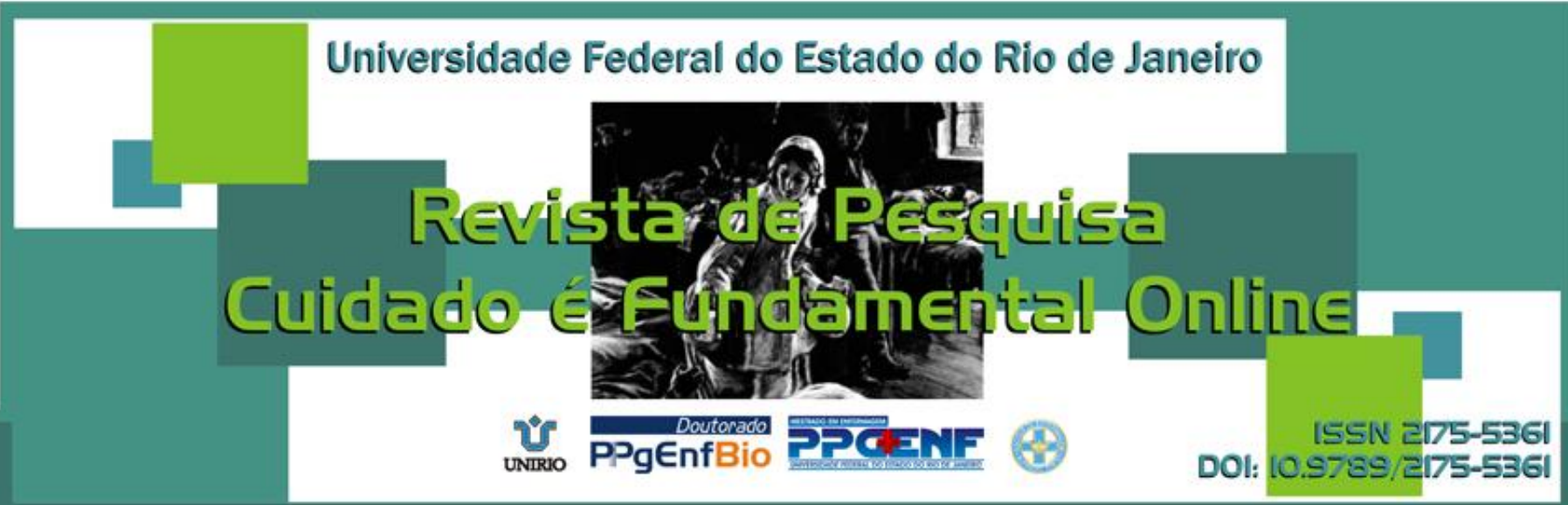

REFELEXÃO

\title{
Nursing theories and models that enhance professional practice
}

Teorias de enfermagem e modelos que fortalecem a prática profissional

Teoría de enfermería y modelos que fortalecen la práctica profesional

Adrize Rutz Porto ${ }^{1}$, Maira Buss Thofehrn ${ }^{2}$, Daiane Dal Pai ${ }^{3}$, Simone Coelho Amestoy ${ }^{4}$, Leandro Rauber Joner ${ }^{5}$, Josiane Santos Palma ${ }^{6}$

ABSTRACT

Objective: The present study aims to propose a reflective rethink theories about nursing from the Theory of Professional Links. Method: This is a theoretical reflection that addresses the importance of theories, as a working tool, which formalize the actions of the nursing profession and as a social practice, which builds daily. Results: First, there is the context of the construction of nursing knowledge and subsequently the Theory of Professional Links as an important management tool working to develop and strengthen human relationships nursing teams, using a model flexible and adaptable to reality and creativity of nurses. Conclusion: It is believed that nursing can provide their professional identity and its body of knowledge, more broadly, including subsidies brought there by the Theory of Professional Links relational issues that mark with a lot of intensity to do nursing. Descriptors: Nursing, Nursing theories, Work environment.

\section{RESUMO}

Objetivo: 0 presente estudo reflexivo tem por objetivo propor um repensar acerca das teorias de enfermagem a partir da Teoria dos Vínculos Profissionais. Método: Trata-se de uma reflexão teórica que aborda a importância das teorias, como instrumentos de trabalho, os quais formalizam as ações da profissão e a enfermagem, como uma prática social, que é cotidianamente construída. Resultados: Primeiramente, destaca-se o contexto da construção do conhecimento da enfermagem e, posteriormente, a Teoria dos Vínculos Profissionais, como um importante instrumento gerencial de trabalho, para desenvolver e fortalecer as relações humanas das equipes de enfermagem, por meio de um modelo flexível e adaptável à realidade e à criatividade dos enfermeiros. Conclusão: Acredita-se que a enfermagem poderá constituir sua identidade profissional e seu corpo de conhecimento, de maneira mais ampla, incluindo-se aí os subsídios trazidos pela Teoria dos Vínculos Profissionais às questões relacionais, as quais marcam com muita intensidade o seu fazer da enfermagem. Descritores: Enfermagem, Teorias de enfermagem, Ambiente de trabalho.

\section{RESUUMEM}

Objetivo: El presente estudio tiene como objetivo proponer una reflexión acerca de repensar las teorías de enfermería de la Teoría de los vínculos profesionales. Método: Se trata de una reflexión teórica que aborda la importancia de las teorías como instrumentos, que formalizan las acciones de la profesión y de enfermería como práctica social, que construye todos los días. Resultados: En primer lugar, se aborda el contexto de la construcción del conocimiento en enfermería y más tarde, la teoría de los vínculos profesionales como importante instrumento gerencial de trabajo para la conformación y fortalecimiento de las relaciones humanas de los equipos de enfermería, a través de una flexible y adaptable a la realidad y la creatividad de las enfermeras. Conclusión: Se cree que la enfermería puede proporcionar su identidad profesional y el cuerpo de conocimiento, en términos más generales, incluidas las subvenciones llevado allí por la Teoría de vínculos profesionales con los temas que marcan con gran intensidad para hacer enfermería. Descriptores: Enfermería, Teoría de enfermería, Ambiente de trabajo.

${ }^{1}$ Enfermeira. Mestre pelo Programa de Pós-Graduação em Enfermagem (PPGEnf) da Universidade Federal de Pelotas (UFPel). E-mail: adrizeporto@gmail.com. ${ }^{2}$ Enfermeira. Pós-doutora na Universidad de Murcia/Espanha. Professora Adjunta do Departamento de Enfermagem da UFPel. E-mail: mairabusst@hotmail.com. ${ }^{3}$ Enfermeira. Doutora em Enfermagem pela Universidade Federal do Rio Grande do Sul (UFRGS). Professora na Escola de Enfermagem (UFRGS). E-mail: daiadalpai@yahoo.com.br. ${ }^{4}$ Enfermeira. Doutora em Enfermagem na Universidade Federal de Santa Catarina (UFSC). Professora Adjunta do Departamento de Enfermagem da UFPel. E-mail: samestoy@terra.com.br. ${ }^{5}$ Enfermeiro. Residente em Saúde Coletiva da Escola de Saúde Pública de Porto Alegre. E-mail: canigia.joner@hotmail.com. ${ }^{6}$ Enfermeira. Doutoranda em Enfermagem na UFPel. E-mail: josisanpalma@hotmail.com. 


\section{INTRODUCCÃO}

O saber da enfermagem, em parte, corresponde a um conjunto de teorias que respaldam a prática profissional e revelam a ação de cuidados, desenvolvida em resposta a uma necessidade do ser humano e da coletividade. Essa prática da enfermagem pode ser vista como social, sendo exercida por profissionais sobre o objeto de seu fazer, por meio da qual estabelecem relações, aplicando o seu saber, como forma de transformar uma realidade concreta. ${ }^{1}$

Nessa perspectiva, as teorias que são construídas a partir de conceitos, modelos e proposições servem de base para fundamentar as ações práticas, auxiliando e explicando as abordagens realizadas junto ao objeto de trabalho - o ser humano. Na enfermagem, o corpo do ser humano, da pessoa em sofrimento físico, psíquico, social e espiritual, pode ser compreendido como esse objeto, no qual se executa a tarefa profissional da enfermagem. Essa tarefa trata-se do cuidado terapêutico, o qual se configura na ação, que se desenvolve e completa com o ser humano, sendo carregado de valor ético e constituindo-se em um bem necessário às pessoas. ${ }^{2}$

Para entender a constituição do saber teórico da enfermagem, cabe brevemente resgatar alguns acontecimentos históricos da constituição da profissão. A enfermagem, durante muitos anos, descreveu seus procedimentos a partir da prática clínica para, nas primeiras décadas do século XX, sistematizá-los como primeira forma organizada do saber, posteriormente, pela instituição dos princípios científicos, que a fundamentam. Mais recentemente, nas décadas de 1950 e 1960, a preocupação incidiu em refletir mais profundamente sobre a profissão, o seu objeto de trabalho e o referencial teórico pertinente ao cuidar, quando, então, começaram a ser construídas as teorias de enfermagem. ${ }^{3}$

As teorias guardam uma relação recíproca com as ações de enfermagem e, em conjunto no exercício cotidiano do trabalho, constituem o cuidado terapêutico, como objeto epistemológico, que se refere à disciplina da enfermagem, para estruturação da sua prática profissional. ${ }^{4}$

No entanto, ainda se observa na literatura a escassez da produção científica nacional sobre as teorias da enfermagem. Um estudo sobre a temática salienta que apenas 4,6\% das publicações utilizaram teorias de enfermagem, sendo que foram levantados 3.757 artigos, de sete periódicos da área de enfermagem, no período de 1998 a $2007 .^{5}$

Esta proposta de reflexão sobre as teorias focaliza a enfermagem, reconhecendo-a como disciplina, profissão e prática social, por intermédio da formalização de conceitos e teorias, como o instrumental adequado, para direcionar a procura pela especificidade da enfermagem, isto é, limites de atuação em relação a outros profissionais, corpo de conhecimento próprio, autonomia, identidade e visibilidade profissional. $^{6}$

Nessa direção, a Teoria dos Vínculos Profissionais (TVP) constitui-se em uma ferramenta para a enfermagem, a qual visa formar e afirmar continuamente as relações interpessoais da equipe na enfermagem, como uma unidade grupal, para além do agrupamento de pessoas, com potencial para o crescimento e o fortalecimento das práticas de cuidado, por meio do estabelecimento de vínculos profissionais saudáveis. Essa teoria, em sua essência, busca trabalhar o relacionamento interpessoal entre enfermeiro e equipe, para assim estabelecer relações mais terapêuticas. Além disso, apreende os vínculos com o desenvolvimento de dinâmicas grupais originadas dos movimentos que se efetivam em duas esferas de relações, o espaço institucional e o espaço específico da ação assistencial, direcionando a ação da equipe ao exercício do cuidado terapêutico. ${ }^{7}$ 
Frente ao exposto, o presente trabalho trata-se de uma reflexão crítica acerca da importância das teorias de enfermagem, utilizando a TVP, como exemplo, para afirmar as teorias, como instrumentos de trabalho, os quais formalizam as ações da profissão e a enfermagem como uma prática social que é cotidianamente construída.

\section{RESULTADOS E DISCUSSÃO}

\section{Contexto epistemológico da enfermagem}

A construção do conhecimento na enfermagem encontra-se ainda em fase inicial, entretanto, a designação do cuidar está baseada no princípio de se tratar de uma prática que se desenvolve sobre a interação enfermeiro/cliente, com a intenção de contribuir para o bem-estar deste último no seu cotidiano. ${ }^{8}$ o cuidado é concebido, por diversos estudiosos, como objeto epistemológico da enfermagem, por envolver o ser humano em sua complexidade, sendo esse o foco do seu trabalho. ${ }^{4}$

O progresso pode também ser visualizado na evolução de concepções a respeito do vocábulo "ser humano" e na mudança paradigmática na atenção e na gestão dispensada às carências de saúde das pessoas. No século XX, houve a afirmação da fragmentação do ser humano em corpo e mente, a partir do modelo biomédico que produz o mecanicismo do fazer, a visão biológica do indivíduo, a especialização sobre o corpo da pessoa, a valorização dos procedimentos técnicos do ato médico e a ênfase na medicina curativa, os quais reforçam a divisão da pessoa em partes, dificultando a união desses fragmentos para uma visão global do ser humano, principalmente, considerando além dos aspectos orgânicos, como a doença, as demais dimensões da vida humana, a saber: psicológica, social, espiritual, econômica, dentre outras.

Por outro lado, o modelo biopsicossocial de atenção e gestão em saúde surgiu como forma de atender às lacunas do modelo antecedente, tais como: a produção de saúde em pessoas portadoras de patologias crônicas, autoimunes, casos sem diagnósticos clínicos ou difíceis de resolver. Ademais, frente a esse recente paradigma, outros saberes profissionais passaram a tornar-se necessários à complexidade que envolve os cuidados de saúde às pessoas. 9

$\mathrm{Na}$ contemporaneidade, grande parte da demanda dos serviços de saúde, em termos de forças profissionais de trabalho, está relacionada a conhecimentos que extrapolam a abordagem do modelo biomédico, exigindo maior abrangência acerca do processo de saúde e de doença, com ênfase nas políticas de saúde e na operação de novos modos de cuidar. Esta questão está atrelada ao fato de que nas últimas décadas têm ocorrido mudanças no perfil clínico e epidemiológico da população, na expectativa de vida, na economia e na política mundial, com consequente reestruturação do setor saúde, impondo novos desafios aos profissionais, os quais devem, de forma articulada, se organizar para enfrentar os problemas que surgem nos diversos cenários de atuação e que repercutem no campo da saúde e no fazer da enfermagem. ${ }^{10}$

Neste contexto, embora a enfermagem adote o cuidado como seu objeto de atuação, a profissão ainda sofre os efeitos da necessidade de ocupar espaços de poder e desenvolver o enfermeiro como um ser sociopolítico. ${ }^{6}$ Além disso, essa área da saúde lida com a permanente crise de identidade, a falta de um estatuto epistemológico e a dependência teórica no campo das práticas científicas da área da saúde e de outras áreas.

Desta forma, questiona-se: por que não pensar o cuidado terapêutico como objeto epistemológico da enfermagem ${ }^{11}$ Ousa-se conceber esse como metaparadigma deste campo do saber 
e, concomitantemente, a enfermagem alicerçada em uma estrutura epistêmica multiparadigmática, diante da carência de identidade profissional e de acordo com as necessidades de cuidado do cliente.

A partir desta perspectiva, percebe-se que as teorias brasileiras de enfermagem estão ainda incipientes, frequentemente, sendo desconsideradas e desvalorizadas nos espaços acadêmicos e profissionais.

Essas lacunas refletem no corpus da enfermagem, em particular, no âmbito do plano pedagógico e assistencial, pois se tratam de um aspecto condicional melhor entendido em relação às teorias e práticas que, depois de importadas, se não forem questionadas, se tornaram majoritárias, ou hegemônicas, porque foram assumidas como medidas salvadoras de dificuldades profissionais acumuladas das experiências históricas do pensar e da ação da enfermagem. No plano da construção científica, observa-se a enfermagem com prejuízos indesejáveis pela falta de poder, como área de conhecimento, face às pressões das políticas de saúde ou pelas instâncias reguladoras dos órgãos de fomento e amparo à pesquisa. ${ }^{12}$

Nas últimas décadas, as mudanças paradigmáticas no campo assistencial e gerencial da saúde vêm ocorrendo rapidamente, desde a construção do processo social, o qual tem como maior conquista o direito à saúde de milhões de brasileiros, a partir de uma base conceitual e filosófica oriunda no movimento da Reforma Sanitária, tendo como marco a XVIII Conferência Nacional de Saúde, realizada em 1986, a qual instituiu sua natureza eminentemente interdisciplinar, devido às múltiplas determinações e mediações históricas. Dessa maneira, aqueles que têm como objeto concreto de trabalho a saúde e a doença no seu âmbito social não podem partir de uma única disciplina para pautar sua ação profissional, porque ele envolve ao mesmo tempo as relações sociais e o social propriamente dito, as expressões emocionais, assim como o biológico, as condições e razões sócio-históricas e culturais de indivíduos e grupos.

Igualmente, no campo pedagógico, as novas Diretrizes Curriculares Nacionais estabelecem uma conquista importante no campo da enfermagem por assinalar, como política, a necessidade de produzir mudanças no processo de formação, propiciando profissionais mais compromissados e engajados com os princípios e diretrizes do Sistema Único de Saúde (SUS). Esses esforços correspondem à preocupação de romper definitivamente com o paradigma biologicista e medicalizante, hospitalocêntrico e centrado nos procedimentos técnicos, atendendo aos novos desafios da contemporaneidade na produção de conhecimentos, na produção das profissões e no estabelecimento de relações saudáveis nas equipes de trabalho.

A vinculação entre educação, trabalho e práticas sociais vislumbra uma nova perspectiva de transformação significativa no cotidiano de cada profissional envolvido com a integralidade da assistência, principalmente, quando organizada a partir das necessidades de saúde da população. Portanto, é neste ponto que a enfermagem, como também as demais profissões da saúde, precisam de maior engajamento, em defesa de uma discussão mais sólida permeada pelos pressupostos da Reforma Sanitária, em uma perspectiva de rever em qual paradigma se situam os modelos atuais de assistência à saúde e qual têm sido seus papéis nesse processo, ação esta que prescinde da reorientação da própria prática profissional. ${ }^{13}$

Partindo desse ponto de vista, cabe salientar que o trabalho em saúde é eminentemente relacional, visto que o seu foco é o cuidado humano, não podendo, como em outros modelos produtivos, ser expresso nos equipamentos ou nos saberes fortemente estruturados, como as tecnologias organizacionais, no entanto, no encontro de subjetividades, comportando um grau de liberdade significativa na escolha do modo de fazer essa produção. ${ }^{14}$ 
É nesse espaço, frequentemente, imperceptível em meio a rotina diária dos diversos trabalhadores de saúde e da discussão da atuação de maneira interdisciplinar, que se concentra a importância das teorias na construção do núcleo específico de conhecimento, da prática social e na consolidação dos limites de atuação da enfermagem, pela sua possibilidade epistemológica no cuidado terapêutico e ética no tratamento das questões profissionais. ${ }^{11}$

\section{Teoria dos Vínculos Profissionais: fortalecimento da prática profissional}

Frente à discussão exposta acerca da construção epistemológica da enfermagem, diante das mudanças multiparadigmáticas da área da saúde, apresenta-se, como uma alternativa, a Teoria dos Vínculos Profissionais (TVP), buscando o fortalecimento da prática profissional, no encontro de intersubjetividades dos sujeitos, os quais atuam, cotidianamente, na mesma equipe.

Dessa forma, o alcance da potencialidade do trabalho em equipe pode ser valorizado à medida que os indivíduos se reconhecem como membros de um grupo, com um objetivo comum. Assim, os integrantes da equipe poderão sentir-se responsáveis, tanto pelos seus fracassos, quanto pelos seus sucessos, participando da tomada de decisão e, principalmente, qualificando a comunicação das informações essenciais para o trabalho da enfermagem.

0 trabalho em equipe pode ser compreendido como "uma rede de relações entre pessoas, poderes, saberes, afetos, interesses e desejos, em que é possível identificar processos grupais, em que trabalhar em equipe equivale a se relacionar".

Nesse sentido, a TVP tem por objetivo dispor ao enfermeiro um modelo de trabalho em equipe na enfermagem que favoreça as relações interpessoais, ou seja, os vínculos profissionais, para o alcance do objeto epistemológico da enfermagem - o cuidado terapêutico -, o qual deve ser comprometido com as pessoas que necessitam de atendimento dos serviços de saúde. Além disso, possibilita o estabelecimento de maior inclusão e significação do grupo na instituição e, ainda, reforça a própria identidade do enfermeiro enquanto líder, isto é, coordenador da equipe e do planejamento de suas atividades.

As pressuposições básicas dessa teoria são baseadas em um olhar filosófico materialista histórico e dialético, em que os vínculos profissionais têm uma natureza expressa em ação e discurso, isto é, os vínculos entre os seres humanos são estabelecidos pela totalidade da pessoa, por meio de sua relação interna e externa, refletida por meio de seus atos e suas palavras, não por uma parte dela, pois o trabalhador não deve separar sua vida interior de sua exterioridade, já que, à medida que os vínculos profissionais se formam, o grupo adquire uma personalidade, uma identidade própria, determinando a sua visualização e valorização na instituição.

Ao abordar as habilidades relacionais do processo gerencial na enfermagem, a TVP apresenta diversos conjuntos de conceitos, especialmente, no que se refere ao sujeito da teoria, o enfermeiro e a ferramenta mediadora entre o enfermeiro e os demais membros da equipe, técnicos e auxiliares de enfermagem e agentes comunitários de saúde, isto é, o modelo para o trabalho em equipe na enfermagem. A meta dessa proposta é voltada para as ações gerenciais e relacionais do enfermeiro, como um mediador no processo de estabelecimento dos vínculos profissionais.

A teoria é um conhecimento de processo, pois ela inclui o processo de trabalho na enfermagem e a interação enfermeiro/equipe na dinâmica grupal. É uma sugestão com alto nível de adaptação à realidade do grupo, porém, é construída pelo método operacional, o qual apresenta as estratégias para implantação da teoria em pauta de forma clara e prática, exigindo que o profissional tenha conhecimento científico, sensibilidade, criatividade, imaginação e habilidades para executá-las. 


\section{CONCLUSÃO}

Esta reflexão crítica que focalizou a importância das teorias de enfermagem para o fortalecimento da profissão e prática social da enfermagem, ao interrelacionar o contexto do conhecimento da enfermagem, destaca a TVP como um modelo a ser experimentado no cotidiano do cuidado, a fim de qualificar as relações humanas no trabalho. Trata-se, contudo, de um modelo flexível e adaptável à realidade e à criatividade dos enfermeiros.

Com base nos modelos teóricos, acredita-se que a enfermagem poderá constituir sua identidade profissional e seu corpo de conhecimento de maneira mais ampla, incluindo-se os subsídios trazidos pela TVP às questões relacionais, as quais marcam com muita intensidade o seu fazer. Este, por sua vez, embora, ao mesmo tempo, necessite de rotinas sistematizadas em operações, deve recusar intervenções mecanizadas, já que o seu objeto de trabalho é o ser humano em sua singularidade e subjetividade, exigindo, portanto, a humanização dos relacionamentos com vistas ao cuidado terapêutico.

Alerta-se para a necessidade da discussão quanto à construção do conhecimento de enfermagem e reforça-se a sugestão de alguns pesquisadores: de que o objeto epistemológico da enfermagem deve ser o cuidado, assim como se tornam imperativos os investimentos e valorização das teorias brasileiras de enfermagem, para obter-se a visibilidade epistemológica e profissional na enfermagem.

\section{REFEREENCIAS}

1. Trezza MCAF, Santos RM, Leite JL. Enfermagem como prática social: um exercício de reflexão. Rev Bras Enferm, Brasília 2008 Nov-Dez; 61(6):904-8.

2. Prado ML, Reibnitz KS. Salud y globalización: retos futuros para el cuidado de enfermería. Investigación Educ Enferm. 2004 Set; 22(2):112-9.

3. Souza MF. Teorias de enfermagem: importância para a profissão. Acta paul. Enferm. 1988 Set; $1(3): 63-5$.

4. Leopardi MT, Gelbcke FL, Ramos FRS. Cuidado: objeto de trabalho ou objeto epistemológico da enfermagem? Texto Contexto Enferm. 2001 Jan-Abr; 10(1):32-49.

5. Schaurich D, Crossetti MGO. Produção do conhecimento sobre teorias de enfermagem: análise de periódicos da área, 1998-2007. Esc Anna Nery Rev Enferm 2010 Jan-Mar; 14(1):182-88.

6. Pai DD, Schrank G, Pedro ENR. O enfermeiro como ser sócio-político: refletindo a visibilidade da profissão do cuidado. Acta paul. enferm. 2006; 19(1):82-7.

7. Thofehrn MB, Leopadi MT. Teoria dos Vínculos Profissionais: formação de um grupo de trabalho. Pelotas: Editora Universitária UFPel; 2009.

8. Basto, ML. Investigação sobre o cuidar de enfermagem e a construção da disciplina: proposta de um percurso. Pensar Enfermagem. 2009; 13(2):11-8.

9. Marco MA. Do modelo biomédico ao modelo biopsicossocial: um projeto de educação permanente. Rev. Bras. Educ. Med. 2006; 30(1):60-72.

10. Tavares CMM. A interdisciplinaridade como requisito para a formação da enfermeira psiquiátrica na perspectiva da atenção psicossocial. Texto Contexto Enferm. 2005; 14(3):403-10. 
11. Thofehrn MB, Leopardi MT. Teorias de enfermagem, trabalho e conhecimento contemporâneo. Texto \& contexto enferm. 2002; 11(1):86-104.

12. Carvalho V. Acerca da interdisciplinaridade: aspectos epistemológicos e implicações para a enfermagem. Rev. Esc. Enferm. USP. 2007; 41(3):500-7.

13. Oliveira TRB. Interdisciplinaridade: um desafio para a atenção integral à saúde. Rev.Saúde.Com. 2007; 3(1):20-7.

14. Neves CAB. Saúde: a cartografia do trabalho vivo. Cad. Saúde Pública. 2008; 24(8):1953-5.

15. Fortuna $C M$ et al. $O$ trabalho de equipe no programa de saúde da família: reflexões a partir de conceitos do processo grupal e de grupos operativos. Rev. Latino-am Enferm. 2005; 13(2):262-8.

Recebido em: 08/10/2011

Revisầo requerida: 21/03/2013

Aprovado em:03/10/2013

Publicado em: 01/12/2013
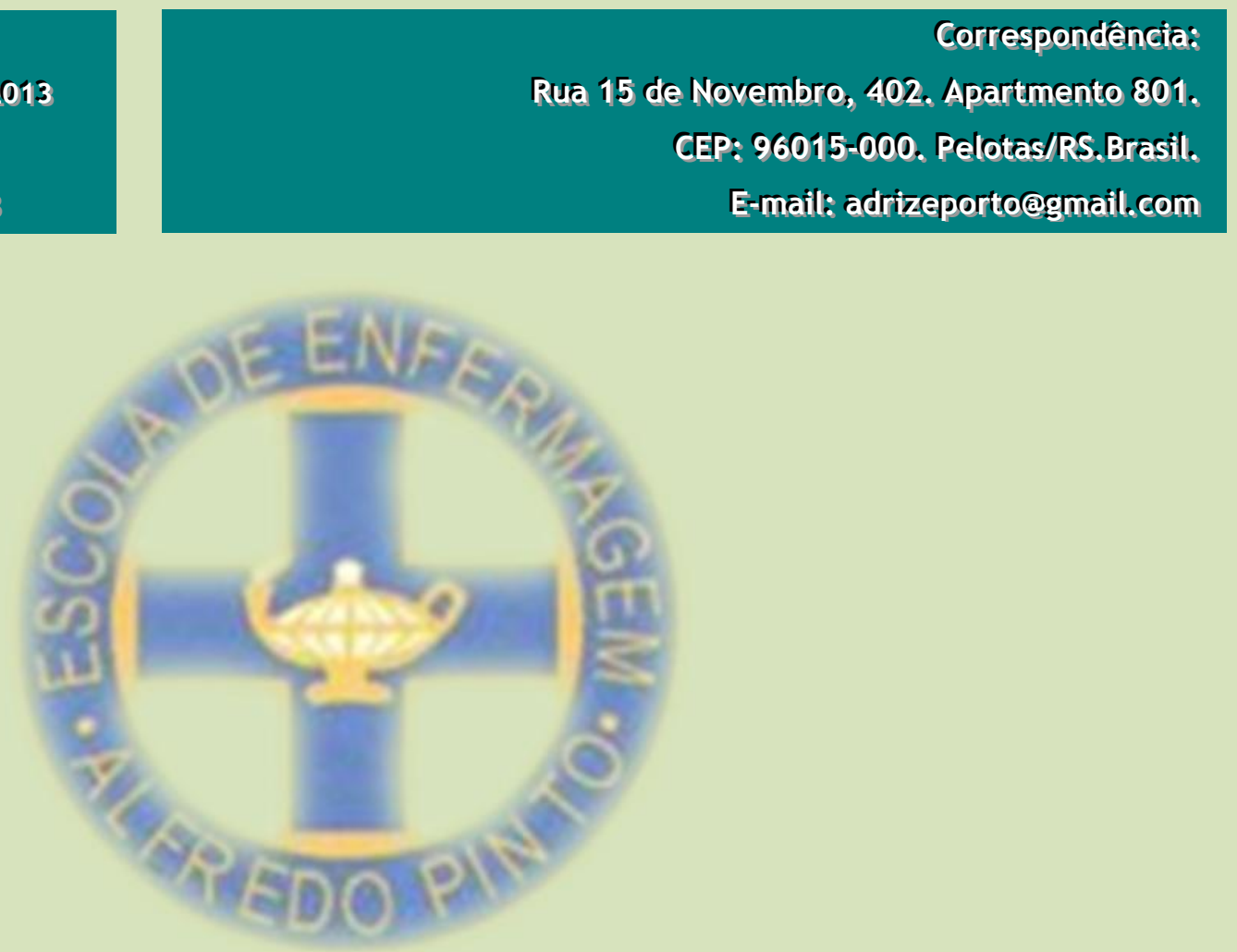